2005

\title{
Laser Induced Fluorescence In A Pulsed Argon Plasma
}

Earl Scime

Costel Biloiu

Christopher Compton

Forrest Doss

Daniel Venture

See next page for additional authors

Follow this and additional works at: https://researchrepository.wvu.edu/faculty_publications

\section{Digital Commons Citation}

Scime, Earl; Biloiu, Costel; Compton, Christopher; Doss, Forrest; Venture, Daniel; Heard, John; Choueiri, Edgar; and Spektor, Rostislav, "Laser Induced Fluorescence In A Pulsed Argon Plasma" (2005). Faculty Scholarship. 578.

https://researchrepository.wvu.edu/faculty_publications/578 


\section{Authors}

Earl Scime, Costel Biloiu, Christopher Compton, Forrest Doss, Daniel Venture, John Heard, Edgar Choueiri, and Rostislav Spektor 


\title{
Laser induced fluorescence in a pulsed argon plasma
}

\author{
Earl Scime, ${ }^{\text {a) }}$ Costel Biloiu, Christopher Compton, Forrest Doss, and Daniel Venture \\ Department of Physics, West Virginia University, P. O. Box 6315, Morgantown, West Virginia 26506-6315 \\ John Heard \\ Department of Physics, 104 Pierce Science Center, Clarion University, Clarion, Pennsylvania 16214 \\ Edgar Choueiri and Rostislav Spektor \\ Department of Mechanical and Aerospace Engineering, Engineering Quad, Princeton University, \\ Princeton, New Jersey 08544
}

(Received 8 October 2004; accepted 24 November 2004; published online 7 January 2005)

\begin{abstract}
A time-resolved laser induced fluorescence (LIF) technique for pulsed argon plasmas is described. A low power, tunable diode laser pumps a three level Ar II transition sequence at a wavelength of $668.6138 \mathrm{~nm}$. With a standard LIF system designed for steady-state plasmas (e.g., $4 \mathrm{kHz}$ optical chopper, $20 \mathrm{kHz}$ band-width detector, and a lock-in amplifier), we demonstrate that the evolution of the ion velocity distribution can be resolved with a time resolution of $1 \mathrm{~ms}$ through a combination of time-series averaging and post-acquisition digital signal processing. (C) 2005 American Institute of Physics. [DOI: 10.1063/1.1848491]
\end{abstract}

Applied to the diagnosis of plasmas three decades ago by Stern and Johnson, ${ }^{1}$ laser induced fluorescence (LIF) has evolved into a powerful tool capable of providing nonintrusive, high resolution, spatially resolved, measurements of particle (ions, atoms, radicals, molecules) velocity distributions in many types of plasma discharges and for a wide range of experimental conditions. ${ }^{2-5}$ Weak LIF emission from a steady-state plasma can be detected in the presence of intense background light by modulating the probing laser beam and employing phase synchronous detection, i.e., using a lock-in amplifier. Pulsed plasmas present the additional challenge of requiring time resolution while still detecting weak LIF emission. The minimum time resolution of a LIF measurement is set by the lifetime of the upper quantum level of the pumped transition, usually on the order of a few nanoseconds. In practice, however, time resolution is limited by the need to collect a sufficient number of LIF emission photons for reasonable signal to noise, the $R C$ time constants of cables, the signal acquisition time requirements of the available electronics, and the particular plasma conditions.

When the properties of the measurement electronics are well known, it is possible to improve the time resolution of LIF measurements by reducing the distorting effects of the lock-in amplifier by digital signal processing. ${ }^{6,7}$ If better time resolution is required, more sophisticated signal acquisition methods must be employed ${ }^{8-10}$ In this work, we demonstrate that it is possible to obtain time and velocity resolved laser induced fluorescence measurements of a pulsed helicon plasma source using a low power, tunable, diode laser. The only modification to the experimental apparatus we typically use for LIF measurements in steady-state plasmas is the addition of a digital oscilloscope on which the LIF emission versus time is averaged over many hundreds of pulses. Time resolution of $1 \mathrm{~ms}$ is sufficient to investigate ion heating by pulsed beat-wave heating in helicon sources ${ }^{11}$ and formation

\footnotetext{
${ }^{a)}$ Electronic mail: escime@wvu.edu
}

of ion beams in pulsed expanding helicon source plasmas. ${ }^{12}$ We note that other researchers have used similar techniques to investigate externally imposed, repetitive, transient phenomena in a steady-state background plasma, e.g., the interaction between ions and ion cyclotron waves in argon plasmas. ${ }^{13}$ However, those experiments employed much more powerful ring dye lasers and only examined the timeaveraged interaction of the ions with the waves. What makes the diagnostic method described here unique is the implementation of these methods with a low power, compact, relatively inexpensive, tunable diode laser ${ }^{14}$ while retaining the capability for time and velocity resolved measurements during transient phenomena.

A velocity resolved, three-level LIF measurement begins with illumination of a collection of particles (ions, atoms, radicals, molecules) with a laser whose bandwidth is much narrower than the particle's absorption linewidth. If the incident photon has an appropriate frequency in the particle's rest frame, then a particular quantum level is optically pumped and the population of an upper quantum level increases. The upper level then decays spontaneously to a third level with a large branching ratio by emitting a photon. The intensity of the fluorescence radiation as a function of laser frequency is a direct measurement of the particle velocity distribution (although processes such as Zeeman splitting may have to be included in the analysis of the data to determine the parent velocity distribution). To obtain nonperturbing measurements, the pumping laser should be operated in the linear regime, i.e., the LIF signal is proportional to the laser intensity. For a Maxwellian distribution of particle velocities, the bulk flow velocity is obtained from the Doppler shift in the fluorescence spectrum. If Doppler broadening dominates over other line-broadening mechanisms, the temperature of the particle ensemble is inferred from the full width at half maximum of the fluorescence line. For LIF studies in pulsed argon plasmas we selected a three level argon LIF scheme first proposed by Severn et al. ${ }^{15}$ 


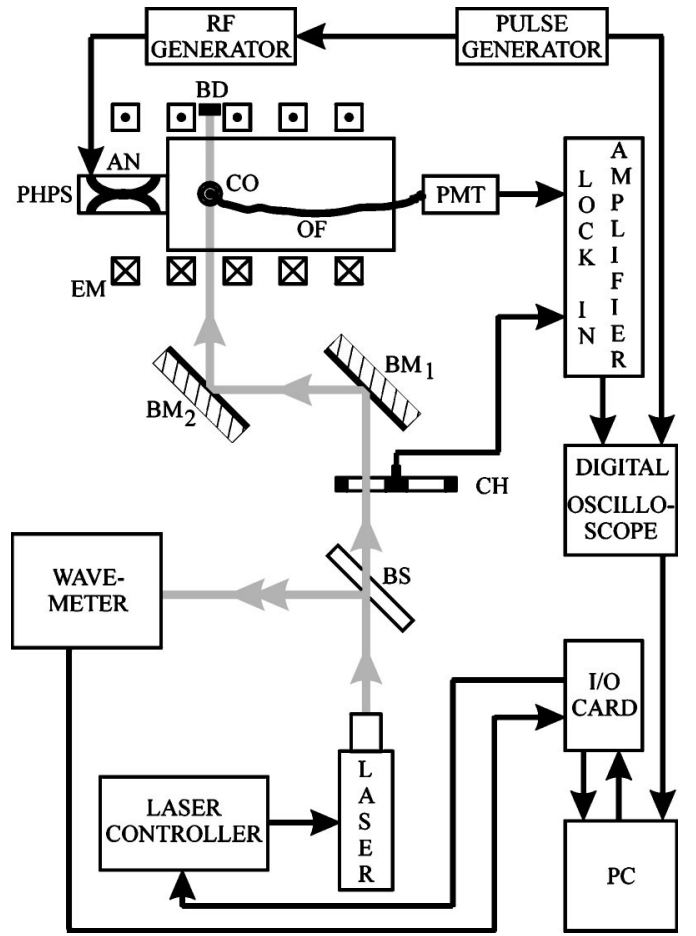

FIG. 1. Experimental setup for time resolved LIF diagnostic: PHPS - pulsed helicon plasma source, AN - antenna, EM - electromagnets, BD - beam dump, CO - collection optics, OF - optical fiber, PMT - photomultiplier tube, $\mathrm{BM}_{1,2}$ alignment mirrors, $\mathrm{CH}$ - mechanical chopper, BS - beam splitter.

A schematic of the LIF system used for pulsed plasma studies is shown in Fig. 1. The tunable diode laser is a Sacher Lasertechnik model Lynx-TEC100 with an external Littrow cavity comprised of a piezoelectric transducer controller (PZT) grating with a beam correction mirror. This Peltier cooled diode laser is characterized by a $1.5 \mathrm{MHz}$ bandwidth and $17 \mathrm{~mW}$ power output at a current of $85 \mathrm{~mA}$. The diode laser has a coarse tuning range of approximately $8 \mathrm{~nm}$ around the factory set central wavelength $(668 \pm 4) \mathrm{nm}$. The Lynx-TEC100 has also a fine-tuning range of $0.4 \mathrm{~nm}$ (or 300 $\mathrm{GHz}$ ). Fine tuning is accomplished by either changing the voltage on the PZT actuator or the temperature of the cavity. Single mode tuning without mode hops is achieved when the external cavity modes and the dispersion bandwidth shift in unison. The $8 \mathrm{GHz}$ mode hop free range of the LynxTEC100 diode laser is large enough to span the absorption linewidth for $0.5 \mathrm{eV}$ Ar ions. For hotter ion populations or high speed bulk flows, the range can be extended to a total mode hop free range of approximately $14 \mathrm{GHz}$ by having the laser controller feedback on the diode current. Although the laser power varies during such extended scans, distortion of the measured LIF emission line shape can be corrected by monitoring the laser power with a laser power meter.

After passing through a $10 \%$ beam splitter, the laser is modulated with a mechanical chopper at $4 \mathrm{kHz}$ and steered into the plasma with a pair of alignment mirrors. $10 \%$ of the beam is sent to the wave meter for real-time wavelength measurement. The laser power after the beam splitter is 15 $\mathrm{mW}$. The laser is injected perpendicular to the magnetic field of the helicon plasma helicon plasma source (see Ref. 11) used for these measurements with the laser polarization axis parallel to the magnetic field. Alignment of the laser polar-

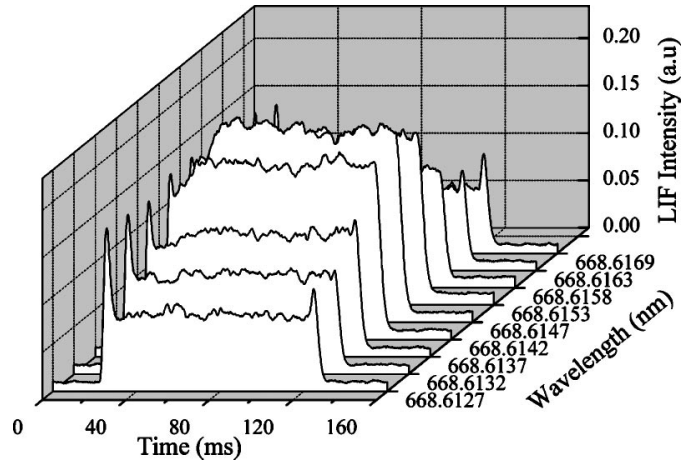

FIG. 2. A set of nine LIF signals averaged over $100 \mathrm{rf}$ pulses without deconvolution. The data are separated by wavelength to highlight the difference in LIF signal as the laser is tuned through the absorption line.

ization axis parallel to the magnetic field avoids pumping the Zeeman split $\sigma$ lines $\left(\Delta M_{J}= \pm 1\right.$ transitions $)$ and the internal Zeeman splitting of the six linearly polarized $\Delta M_{J}=0$ transitions ( $\pi$ lines) is ignorable for the magnetic field strength of the helicon source $(<500 \mathrm{G})$.

The fluorescence radiation is collected at $90^{\circ}$ with respect to the laser beam. The light collection assembly consists of a $10 \mathrm{~cm}$ focal length, 2.54-cm-diameter collection lens followed by a $2.54 \mathrm{~cm}$ diameter, $5.0 \mathrm{~cm}$ focal length focusing lens. The numerical aperture of the focusing lens was chosen to match the numerical aperture $(\mathrm{NA}=0.22)$ of a $200-\mu \mathrm{m}$-diameter fused silica optical fiber. The overlapping 3-mm-diameter laser beam and 5-mm-diameter collection focus spot yield a measurement volume of $60 \mathrm{~mm}^{3}$. Light exiting the collection fiber passes through a $1 \mathrm{~nm}$ bandpass interference filter centered at $442.6 \mathrm{~nm}$. Following the filter is a Hamamatsu HC124-60 PMT detector with an integrated $20 \mathrm{kHz}$ bandwidth preamplifier. A Stanford Research SR 830 lock-in amplifier, referenced to the modulation signal from the mechanical chopper, is used to isolate the LIF signal from background emission at the fluorescence wavelength. The signal from the lock-in amplifier is sent to one channel of a Tektronix TDS 460A digital oscilloscope. Another channel records the pulsed waveform from the function generator used to drive the pulsed helicon discharge. $250 \mathrm{~W}$ of $\mathrm{rf}$ power at $13.56 \mathrm{MHz}$ pulsed at a frequency of $5 \mathrm{~Hz}$ (with a $50 \%$ duty cycle) yields a plasma density of approximately $2 \times 10^{11} \mathrm{~cm}^{-3}$ in the middle of the plasma pulse and an electron temperature of $3.5 \mathrm{eV}$ for a magnetic field strength of $260 \mathrm{G}$ and a neutral pressure of 1 mTorr. ${ }^{11}$ The helicon source consists of 6-cm-diameter, 37-cm-long Pyrex ${ }^{\mathrm{TM}}$ tube mated to a $20-\mathrm{cm}$-diameter, 46-cm-long expansion chamber. The LIF measurements were performed in the expansion chamber, $10 \mathrm{~cm}$ away from the end of the helicon source.

The LIF signal is accumulated during each plasma pulse (and dwell interval) at a digitation rate of $5 \mathrm{kHz}$ and averaged over 100 cycles. After each averaged time series is acquired, the laser is then tuned to a new wavelength. Typically 30 wavelengths over a laser frequency range of $8 \mathrm{GHz}$ are needed to obtain reliable ion distribution function measurements. After subtraction of the time-dependent background signal, the LIF signal to noise is better than 10:1. The time resolution of the measurement is limited by the integration time of the lock-in amplifier and the update rate of the lock-in output electronics. Nine time and velocity resolved 

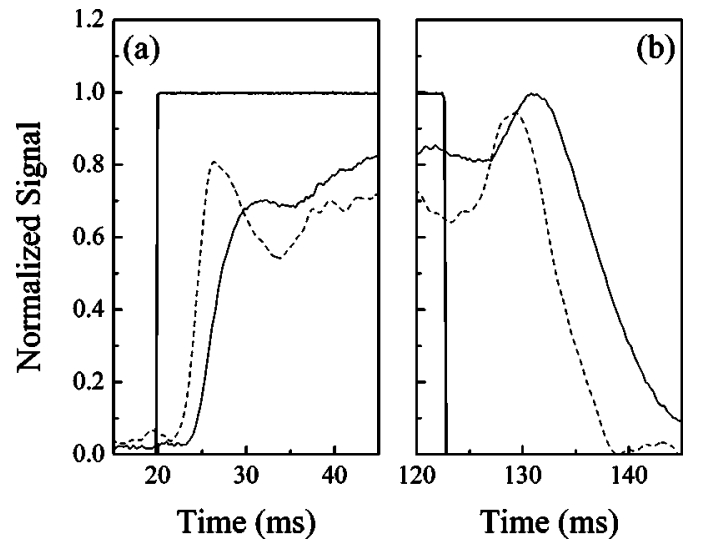

FIG. 3. Expanded view of the beginning (a) and end (b) of the rf pulse amplitude (square solid line), the LIF signal from the lock-in amplifier with a $3 \mathrm{~ms}$ integration time (solid curve), and the LIF signal after deconvolution (dashed curve) of the low pass filtering effects of the lock-in amplifier from the PMT signal as described in Appendix A of Ref. 7.

LIF measurements are plotted in Fig. 2. The lock-in time constant was $1 \mathrm{~ms}$ and the lock-in output signal was updated at a rate of $512 \mathrm{~Hz}$, thereby limiting the time resolution of the data to $1.95 \mathrm{~ms}$. When set to a shorter integration time, the lock-in was unable to differentiate between the spontaneous and induced emission in the plasma. Replacing the mechanical chopper with an acousto-optic modulator operating at higher frequencies than $4 \mathrm{kHz}$ would allow the time resolution to be improved while maintaining the simplicity of the lock-in based detection scheme. However, the substantial reduction in incident laser power that accompanies the use of acousto-optic modulators is problematic for the low power diode laser LIF system used in these experiments. Treating the lock-in integration process as processing by a linear $R C$ low pass filter, improved time resolution is obtainable by digital signal processing of the measured wave forms (see Fig. 3).

Ion velocity distribution measurements at different times during the pulse are obtained by taking temporal slices in the LIF intensity-wavelength plane. Typical individual distribution measurements are shown in Fig. 4 after background subtraction. The perpendicular ion temperature calculated from the full width at half maximum of the Gaussian fits versus time varies from 0.06 to $0.07 \mathrm{eV}$ throughout the discharge. The shift in frequency of the peak LIF emission relative to the rest frame frequency is also relatively constant throughout the discharge (and consistent with a bulk perpendicular ion speed of zero). Note that the plasma afterglow

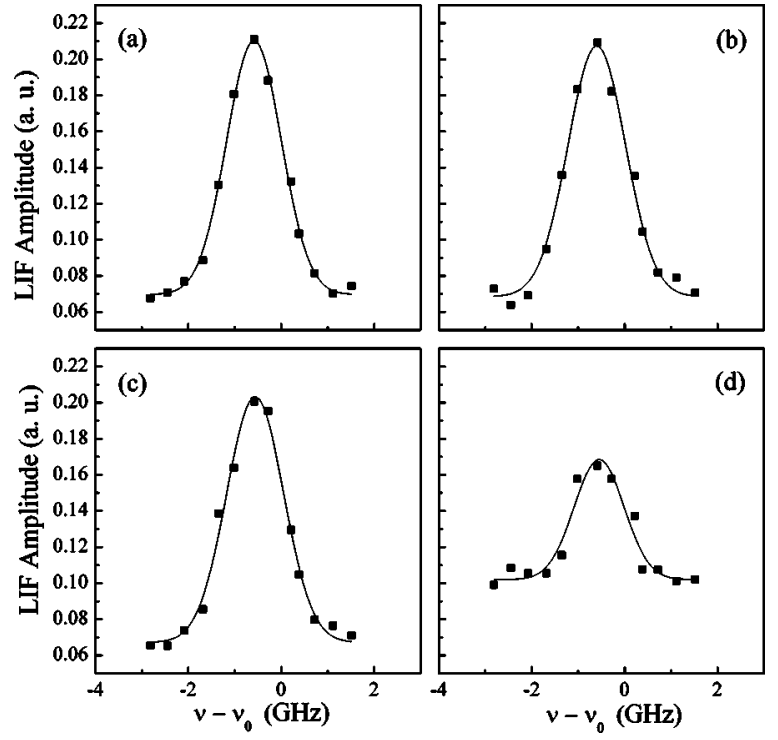

FIG. 4. Ion velocity distribution functions obtained from slices of the time dependent LIF signals during the pulse at: (a) $t=50 \mathrm{~ms}$; (b) $t=75 \mathrm{~ms}$; (c) $t$ $=100 \mathrm{~ms}$; and $(\mathrm{d}) t=125 \mathrm{~ms}$.

lasts for approximately $8 \mathrm{~ms}$ after the rf is turned off. It is possible that the LIF afterglow measurements could be used to investigate both the energy and plasma confinement times of helicon source plasmas.

This work was supported by the U.S. Department of Energy under Grant No. ER45849.

${ }^{1}$ R. A. Stern and J. A. Johnson III, Phys. Rev. Lett. 341548 (1975).

${ }^{2}$ D. H. Hill, S. Fornaca, and M. G. Wickham, Rev. Sci. Instrum. 54, 309 (1983).

${ }^{3}$ D. J. Trevor et al., Appl. Phys. Lett. 57, 1188 (1990).

${ }^{4}$ J. Amorim et al., J. Phys. D 33, R51 (2000).

${ }^{5}$ A. M. Keesee, E. E. Scime, and R. Boivin, Rev. Sci. Instrum. 75, 4091 (2004).

${ }^{6}$ P. A. Jansson, Deconvolution: With Applications in Spectroscopy (Academic, Orlando, 1984).

${ }^{7}$ G. Jackson et al., Spectrochim. Acta, Part B 56, 2449 (2001).

${ }^{8}$ G. Bachet et al., J. Phys. III 6, 1157 (1996).

${ }^{9}$ B. Pelissier and N. Sadeghi, Rev. Sci. Instrum. 67, 3405 (1996).

${ }^{10}$ G. Bachet et al., Phys. Rev. Lett. 80, 3260 (1998).

${ }^{11}$ R. Spektor and E. Choueiri, Phys. Rev. E 69, 046402 (2004).

${ }^{12}$ C. Charles and R. Boswell, Phys. Plasmas 11, 3808 (2004).

${ }^{13}$ M. Sarfaty, S. De Souza-Machado, and F. Skiff, Phys. Plasmas 3, 4316 (1996).

${ }^{14}$ R. F. Boivin and E. E. Scime, Rev. Sci. Instrum. 74, 4352 (2003).

${ }^{15}$ G. D. Severn, D. A. Edrich, and R. McWilliams, Rev. Sci. Instrum. 69, 10 (1998). 